\title{
Direct and Transdentinal (Indirect) Antibacterial Activity of Commercially Available Dental Gel Formulations against Streptococcus mutans
}

\author{
Tamer Tüzüner $^{\mathrm{a}}$ Ayça Tuba Ulusoy ${ }^{c}$ Ozgul Baygin $^{\mathrm{a}}$ Gorkem Yahyaoglu $^{\mathrm{a}}$ \\ Ilkay Yalcin ${ }^{a}$ Kurtulus Buruk ${ }^{b}$ John Nicholson ${ }^{d}$ \\ Departments of a Pediatric Dentistry, Faculty of Dentistry and ${ }^{b}$ Microbiology, Faculty of Medicine, Karadeniz \\ Technical University, Trabzon, and 'Department of Pediatric Dentistry, Ondokuz Mayıs University, Samsun, Turkey; \\ ${ }^{\mathrm{d}}$ Department of Biomaterials Chemistry, University of Greenwich, School of Science, Chatham, UK
}

\section{Key Words}

Antibacterial dental gels $\cdot$ Streptococcus mutans $\cdot$ Cavity disinfectant $\cdot$ Chlorhexidine $\cdot$ Aloe vera $\cdot$ Hyaluronic acid

\begin{abstract}
Objective: To evaluate the direct and transdentinal (indirect) agar diffusion antibacterial activity of different commercially available antibacterial dental gel formulations against Streptococcus mutans. Materials and Methods: The commercially available dental gel formulations were Corsodyl ${ }^{\circledR}$ (COG, $1 \%$ chlorhexidine), Cervitec ${ }^{\circledR}$ (CEG, $0.2 \%$ chlorhexidine $+0.2 \%$ sodium fluoride), Forever Bright ${ }^{\circledR}$ (FOB, aloe vera), Gengigel $^{\circledR}$ (GEG, $0.2 \%$ hyaluronic acid), 35\% phosphoric acid gel and distilled water (control). Direct agar diffusion was performed by isolating three wells from brain-heart infusion agar plates using sterile glass pipettes attached to a vacuum pump and adding $0.1 \mathrm{ml}$ of the gels to each well. Transdentinal (indirect) agar diffusion was performed by applying gel to $0.2-$ and $0.5-\mathrm{mm}$-thick human dentin discs previously etched with phosphoric acid and rinsed with distilled water. Zones formed around the wells and the dentin discs were measured and analyzed using Kruskal-Wallis and MannWhitney $U$ tests with Bonferroni correction $(p<0.01)$. Results: Direct agar diffusion tests showed significant differ-
\end{abstract}

\begin{tabular}{ll}
\hline KARGER & $\begin{array}{l}\text { ( } 2013 \text { S. Karger AG, Basel } \\
1011-7571 / 13 / 0224-0397 \$ 38.00 / 0 \quad \text { Karger }\end{array}$ \\
$\begin{array}{l}\text { E-Mail karger@karger.com } \\
\text { www.karger.com/mpp }\end{array}$ & $\begin{array}{l}\text { This is an Open Access article licensed under the terms of the } \\
\text { Creative Commons Attribution-NonCommercial 3.0 Un- } \\
\text { ported license (CC BY-NC) (www.karger.com/OA-license), } \\
\text { applicable to the online version of the article only. Distribu- } \\
\text { tion permitted for non-commercial purposes only. }\end{array}$
\end{tabular}

ences among all gel formulations $(p<0.01)$ except for COG and CEG ( $p>0.01)$. COG and CEG exhibited higher antibacterial effects compared to FOB and GEG $(p<0.01)$ in both direct and transdentinal (indirect) testing procedures. GEG did not show any antimicrobial activity in transdentinal (indirect) testing. Conclusion: Commercially available dental gels inhibited S. mutans, which may indicate their potential as cavity disinfectants.

Copyright $\odot 2013$ S. Karger AG, Basel

\section{Introduction}

Insufficient removal of caries-affected enamel and dentin represents a serious problem in restorative dentistry, as any bacteria remaining in the cavity after preparation may cause secondary caries [1-3]. The use of an antibacterial material as a cavity disinfectant has been recommended in order to provide an aseptic environment before pulp capping and lining $[4,5]$.

Chlorhexidine digluconate (CHX) possesses a broad spectrum of activity against various oral microorganisms. At high concentrations, CHX can diffuse into bacteria, causing irreversible damage including precipitation and coagulation of cytoplasmic content, and death of the mi- 
Table 1. Details of antibacterial gel formulations

\begin{tabular}{|c|c|c|}
\hline & & Lot No. \\
\hline Corsodyl gel ${ }^{\circledR}$ (COG) & $1 \%$ chlorhexidine gluconate, GlaxoSmithKline, Baranzate, Italy & 1104760 \\
\hline Cervitec gel $^{\circledR}(\mathrm{CEG})$ & $\begin{array}{l}0.2 \% \text { chlorhexidine digluconate }+0.2 \% \text { sodium fluoride, Ivoclar Vivadent, } \\
\text { Schaan, Liechtenstein }\end{array}$ & 16823 \\
\hline Forever Bright/aloe vera toothgel ${ }^{\circledR}(\mathrm{FOB})$ & Aloe barbadensis gel, bee propolis, fluoride-free, Scottsdale, Ariz., USA & 30610 \\
\hline Gengigel $^{\circledR}$ (GEG) & $0.2 \%$ hyaluronic acid, Ricerfarma, Milan, Italy & 1008100 \\
\hline Phosphoric acid gel (PAG) & $35 \%$ phosphoric acid gel, Scotchbond Etchant, 3M ESPE, Seefeld, Germany & N206218 \\
\hline
\end{tabular}

croorganisms $[5,6]$. In an effort to improve dentin and root canal disinfection, researchers have examined the use of CHX-based gel formulations at different concentrations for testing the bioavailability of CHX in the application area and have found antibacterial action against Streptococcus mutans [4, 7-9].

Dentin plays an important role as a physical barrier against the diffusion of bacteria and bacterial by-products towards the pulp [5]. The ideal cavity disinfectant should provide effective antibacterial action without causing toxicity to pulp cells $[5,6]$. However, in addition to the beneficial effects described above, CHX formulations have also been reported to have cytotoxic characteristics [10-12]. A number of studies have recommended less cytotoxic, biocompatible gel formulations in an effort to avoid any potentially harmful effects from CHX [10-12] and provide prolonged antibacterial features in deep dentin cavities [7-9]. Aloe vera gel has been reported to have potential antimicrobial, antioxidant and anti-inflammatory properties [13-15] with low levels of cytotoxicity [14]. A recent study by George et al. [13] utilizing direct agar diffusion testing found aloe vera gel to have antibacterial effects against $S$. mutans, which were attributed to saponins, glycoside-containing soapy molecules that have exhibited cleansing and antiseptic properties. Aloe vera's antibacterial properties have also been attributed to the plant's natural anthraquinones [13-15]. Moreover, it contains bee propolis, which is a brownish resinous substance collected by bees mainly from plants, and this may also contribute antibacterial properties [7, 8, 16, 17]. Hyaluronic acid-based gel formulations have also been found to have antibacterial effects on oral bacteria $[18,19]$ and to help improve wound healing [18]. Moreover, the chemical formulations of these gels have also been found to be biocompatible with odontoblast cells [20,21].

These gels are not designed specifically for cavity disinfection, but are available over the counter as general oral disinfectants. Therefore the hypothesis of this study was to evaluate the efficacy of commercially available gel formulations on $S$. mutans with two relevant in vitro test models.

\section{Materials and Methods}

Details of the antibacterial gel formulations are listed in table 1. Antibacterial activity against $S$. mutans (ATCC 25175; Sigma-Aldrich, St. Louis, France) was evaluated using direct and indirect agar diffusion methods.

\section{Direct Agar Diffusion Testing}

S. mutans was stored at $-20^{\circ} \mathrm{C}$ and cultured on blood agar (Merck, Germany) at $37^{\circ} \mathrm{C}$ for $24 \mathrm{~h}$ in $5 \% \mathrm{CO}_{2}$. A single colony was transferred to brain-heart infusion broth (Merck) and incubated at $37^{\circ} \mathrm{C}$ for $24 \mathrm{~h}$. A suspension was prepared by diluting the broth culture with phosphate-buffered saline to a $0.5 \mathrm{MacF}$ arland standard $\left(1.5 \times 10^{8}\right.$ organisms $\left./ \mathrm{ml}\right)$. A sample of $10 \mu \mathrm{l}$ of the $S . m u$ tans suspension was flood-inoculated onto the surface of brainheart infusion agar plates and then air-dried at $37^{\circ} \mathrm{C}$ for $15 \mathrm{~min}$. For each gel formulation, 3 wells $6 \mathrm{~mm}$ in diameter were separated from the plates using sterile glass pipettes attached to a vacuum pump, and $0.1 \mathrm{ml}$ of the gel samples were introduced into the wells.

\section{Transdentinal (Indirect) Agar Diffusion Testing [5]}

A total of 12 sound human third-molar teeth were collected after obtaining approval from the University Ethics Committee and informed consent from patients. Teeth were mounted in a precision cutting device (Micra Cut 125; Metkon, Turkey) fitted with a water-cooled diamond saw (Diamond Cutting Disc; Dimos$\mathrm{M}$, Turkey). Teeth were sliced transversally to obtain 3 discs from each tooth $(n=36)$. Dentin surfaces of the discs were polished with wet 600 -grit silicon carbide paper to obtain discs measuring 0.2 $\mathrm{mm}$ (18 discs) and $0.5 \mathrm{~mm}$ (18 discs) in thickness and $8 \mathrm{~mm}$ in diameter according to a digital caliper (150 mm Digital Caliper; China). Discs were treated with $0.5 \mathrm{M}$ ethylenediaminetetraacetic acid solution ( $\mathrm{pH}$ 7.2) for $2 \mathrm{~min}$ to remove the smear layer and open the dentin tubules, rinsed twice with sterile deionized water and autoclaved at $121^{\circ} \mathrm{C}$ for $20 \mathrm{~min}$, after which they were randomly distributed among the gel formulations. 
Table 2. Antibacterial effects (inhibition zones) of different gel formulations against S. mutans

\begin{tabular}{|c|c|c|c|}
\hline \multirow{2}{*}{$\begin{array}{l}\text { Gel formulations } \\
(n=3) \text { for each test }\end{array}$} & \multicolumn{3}{|c|}{ Inhibition zones, $\mathrm{mm}$} \\
\hline & $\begin{array}{l}\text { direct agar } \\
\text { diffusion test }\end{array}$ & $\begin{array}{l}\text { transdentinal, indirect } \\
(0.2 \mathrm{~mm} \text { thickness })\end{array}$ & $\begin{array}{l}\text { transdentinal, indirect } \\
(0.5 \mathrm{~mm} \text { thickness })\end{array}$ \\
\hline Corsodyl gel (COG) & $20(20-20)^{\mathrm{A}}$ & $18(17-19)^{\mathrm{A}}$ & $17(17-18)^{\mathrm{A}}$ \\
\hline Cervitec gel (CEG) & $20(20-20)^{\mathrm{A}}$ & $16(16-17)^{\mathrm{B}}$ & $15(15-16)^{\mathrm{B}}$ \\
\hline Forever Bright (FOB) & $15(14-15)^{\mathrm{B}}$ & $14(13-14)^{\mathrm{C}}$ & $13(12-13)^{\mathrm{C}}$ \\
\hline Gengigel (GEG) & $12(12-12)^{\mathrm{C}}$ & $0(0-0)^{\mathrm{D}}$ & $0(0-0)^{\mathrm{D}}$ \\
\hline Phosphoric acid gel (PAG) & $31(30-31)^{\mathrm{D}}$ & $0(0-0)^{\mathrm{D}}$ & $0(0-0)^{\mathrm{D}}$ \\
\hline Control, sterile (CNT) distilled water & $0(0-0)^{\mathrm{E}}$ & $0(0-0)^{\mathrm{D}}$ & $0(0-0)^{\mathrm{D}}$ \\
\hline
\end{tabular}

Data are expressed as median and range in parentheses. In each column, different superscript capital letters indicate significant differences $(\mathrm{p}<0.01)$, whereas the same letters indicate no significant differences among the groups $(\mathrm{p}>0.01)$.

Prior to gel application, discs were placed in a sterile metallic tray and etched with 35\% phosphoric acid gel (PAG, Scotchbond Etchant, 3M ESPE, Seefeld, Germany) for $15 \mathrm{~s}$, rinsed and dried in order to mimic the clinical situation. Gels were applied to the entire occlusal side of each disc and left for 1 min before removing with sterile paper. Discs were placed in Petri dishes, prepared as described above, with the pulpal side of the disc facing the agar, and incubated at $37^{\circ} \mathrm{C}$ for $24 \mathrm{~h}$ in $5 \% \mathrm{CO}_{2}$.

After $24 \mathrm{~h}$, both for direct and transdentinal (indirect) agar diffusion testing, the inhibition zones in millimeters using a digital caliper (150 mm Digital Caliper) were measured once by 2 independent observers (blinded to the gel formulations) taken at the greatest distance between two points at the outer limit of the inhibition halo formed around the well and discs. Measurements were repeated 3 times to ensure the homogeneity of findings.

\section{Statistical Analysis}

Data analysis was performed using the Statistical Packages for Social Sciences for Windows 15.0 (SPSS Inc., Chicago, Ill., USA). The kappa value was calculated in order to measure the level of agreement between observers. The nonparametric Kruskal-Wallis and Mann-Whitney $U$ test with Bonferroni correction $(\mathrm{p}<0.01)$ were used to compare significant differences among groups.

\section{Results}

The antibacterial activity of the different gels tested against $S$. mutans is shown in table 2. The kappa value was 0.95 between the 2 independent observers.

No inhibition zone was observed in the control gel formulation using either direct or transdentinal (indirect) agar diffusion testing. No inhibition zone was observed for the PAG group using transdentinal (indirect) testing; however, with direct testing, PAG showed significantly larger inhibition zones than all other groups tested (Cor- sodyl gel ${ }^{\circledR}, \mathrm{COG}, \mathrm{p}=0.002$; Cervitec gel ${ }^{\circledR}, \mathrm{CEG}, \mathrm{p}=0.002$; Forever Bright ${ }^{\circledR}$, FOB, $p=0.003$; Gengigel ${ }^{\circledR}$, GEG, $p=$ 0.002; control, $\mathrm{p}=0.002$ ). The Mann-Whitney $U$ test and Bonferroni correction showed COG and CEG to have similar $(\mathrm{p}=1.000)$ antibacterial effects on $S$. mutans based on direct testing. COG and CEG also resulted in higher antibacterial effects compared to FOB $(p=0.002)$ and GEG $(p=0.002)$ in direct testing. All of the commercially available dental gel formulations exhibited antibacterial effects against $S$. mutans, with the exception of GEG, when tested using the transdentinal (indirect) method. Based on transdentinal (indirect) testing, the antibacterial effects of COG were significantly higher than those of CEG (0.2 mm, $\mathrm{p}=0.008 ; 0.5 \mathrm{~mm}, \mathrm{p}=0.003)$. Both COG $(\mathrm{p}=0.003)$ and CEG $(\mathrm{p}=0.002)$ also exhibited significantly higher antibacterial effects compared to $\mathrm{FOB}$ at 0.2 and $0.5 \mathrm{~mm}$ dentin thickness.

\section{Discussion}

Direct agar diffusion test results showed that all commercially available dental gel formulations tested in this study exhibited antibacterial effects against S. mutans. The transdentinal (indirect) agar diffusion activity tests using dentin thicknesses of 0.2 and $0.5 \mathrm{~mm}$ showed that COG gel had the greatest antibacterial effects, followed by CEG and FOB, whereas GEG and PAG did not show any antibacterial effects against $S$. mutans. The control group did not exhibit any antimicrobial action using both testing (direct or indirect) methods.

The results of this study showed that all commercially available antibacterial dental gel formulations exhibited 
direct antibacterial activity against $S$. mutans bacteria. Previous reports have also shown that higher CHX concentrations are better at inhibiting bacteria than lower concentrations $[5,22,23]$, but with an increased risk of cytotoxicity $[6,10,11]$. In the present study, CEG and COG exhibited similar antibacterial effects $(p>0.01)$ according to direct test results. Although the $\mathrm{CHX}$ concentration of CEG $(0.2 \%)$ is lower than that of COG $(1 \%)$, the $0.5 \%$ fluoride content of CEG could be responsible for its acceptable antibacterial activity according to the direct agar diffusion test results. Moreover, the antibacterial effects of FOB and GEG were found to be significantly lower $(p<0.01)$ than those of both CHX gel formulations in direct testing. The findings in this study regarding the antibacterial effects of FOB on S. mutans bacteria are similar to those of one recent study [13]. The findings in this study regarding the weaker antibacterial effects of GEG when compared to the $\mathrm{CHX}$ formulations are also similar to those of another recent study using streptococci [19].

In the transdentinal (indirect) test method used in this study, PAG was applied and removed by rinsing prior to gel application in order to mimic dental adhesive application in a clinical situation. Contrary to results of direct agar diffusion testing, PAG showed no inhibitory effects against $S$. mutans in transdentinal (indirect) testing. This finding was similar to that of a previous report [5] and shows that the buffering capacity of dentin decreases the antibacterial effects of PAG, particularly in the transdentinal (indirect) test method. Furthermore, our results indicate that if phosphoric acid was unable to exert any antibacterial action in transdentinal (indirect) testing, the observed antibacterial effects would have been related strictly to the composition of the gel formulations, which were found to be: COG > CEG > FOB $(\mathrm{p}<0.01)$.

All of these direct and transdentinal (indirect) findings indicate that the gel formulations tested may have potential as cavity disinfectants once their inhibitor effects on the microorganisms associated with secondary caries have been tested.

When comparing the antibacterial effects of gel formulations in direct versus transdentinal (indirect) testing, it could be clearly demonstrated that all of the gel formulations exerted higher antibacterial effects in direct testing compared to transdentinal (indirect) testing. Moreover, COG, CEG and FOB showed sufficient antibacterial effects after a short, 1-min application period that induced transdentinal (indirect) activity for up to 24 $h$. This finding indicates that FOB should also be considered an acceptable material with respect to the dentin thicknesses tested $(0.2$ and $0.5 \mathrm{~mm})$. However, GEG was unable to inhibit bacterial growth in transdentinal (indirect) testing, regardless of dentin thickness. It is possible that the hyaluronic acid molecules did not have an opportunity to exhibit sufficient transdentinal (indirect) activity due to their random diffusion in the GEG matrix. Alternatively, this finding could also be explained by the inability of sufficient amounts of hyaluronic acid to diffuse across the dentin substrate in the short application (1-min) period. Overall, the present study found FOB and GEG to have beneficial antibacterial activity against $S$. mutans that was similar to that of CHX formulations (COG and CEG). However, further studies regarding the antibacterial effects of these gel formulations on other bacteria such as lactobacillus or Actinomyces and the transdentinal cytotoxic effects of FOB and GEG formulations are required in order to understand the ideal conditions for healing of the pulp-dentin complex. In view of the potential direct and transdentinal (indirect) antibacterial effects of FOB and the direct effects of GEG, future studies should continue to explore the use of these formulations as alternatives to $\mathrm{CHX}$, given their relatively lower cytotoxicity.

\section{Conclusions}

CHX formulations (COG and CEG) showed significantly higher antibacterial activity when compared to FOB and GEG. All commercially available gels that were tested could be antibacterial formulations against $S$. $m u$ tans.

References

1 Al-Samhan A, Al-Enezi H, Alomari Q: Clinical evaluation of posterior resin composite restorations placed by dental students of $\mathrm{Ku}$ wait University. Med Princ Pract 2010;19: 299-304.

2 Al-Omari WM, Al-Omari QD, Omar R: Effect of cavity disinfection on postoperative sensitivity associated with amalgam restorations. Oper Dent 2006;31:165-170.

-3 Mobarak EH, Shabayek MM, Mulder J, et al: Caries experience of Egyptian adolescents: does the atraumatic restorative treatment approach offer a solution? Med Princ Pract 2011;20:545-549.

-4 Ergücü Z, Hiller KA, Schmalz G: Influence of dentin on the effectiveness of antibacterial agents. J Endod 2005;31:124-129.

$\checkmark 5$ Lessa FC, Nogueira I, Vargas F da S, et al: Direct and transdentinal antibacterial activity of chlorhexidine. Am J Dent 2010;23:255-259. 
-6 Hidalgo E, Dominguez C: Mechanisms underlying chlorhexidine-induced cytotoxicity. Toxicol In Vitro 2001;15:271-276.

7 Kandaswamy D, Venkateshbabu N, Gogulnath $\mathrm{D}$, et al: Dentinal tubule disinfection with $2 \%$ chlorhexidine gel, propolis, morinda citrifolia juice, $2 \%$ povidone iodine, and calcium hydroxide. Int Endod J 2010;43:419423.

8 Valera MC, da Rosa JA, Maekawa LE, et al: Action of propolis and medications against Escherichia coli and endodoxin in root canals. Oral Surg Oral Med Oral Pathol Oral Radiol Endod 2010;110:70-74.

-9 Signoretti FG, Gomes BP, Montagner F, et al: Influence of $2 \%$ chlorhexidine gel on calcium hydroxide ionic dissociation and its ability of reducing endotoxin. Oral Surg Oral Med Oral Pathol Oral Radiol Endod 2011;111:653-658.

10 Faria G, Celes MR, De Rossi A, et al: Evaluation of chlorhexidine toxicity injected in the paw of mice and added to cultured L929 fibroblasts. J Endod 2007;33:715-722.

$\checkmark 11$ Lessa FC, Aranha AM, Nogueira I, et al: Toxicity of chlorhexidine on odontoblast-like cells. J Appl Oral Sci 2010;8:50-58.
12 Lessa FC, Nogueira I, Huck C, et al: Transdentinal cytotoxic effects of different concentrations of chlorhexidine gel applied on acidconditioned dentin substrate. J Biomed Mater Res B Appl Biomater 2010;92:40-47.

13 George D, Bhat SS, Antony B: Comparative evaluation of the antimicrobial efficacy of aloe vera tooth gel and two popular commercial toothpastes: an in vitro study. Gen Dent 2009 57:238-241.

14 Wynn RL: Aloe vera gel: update for dentistry. Gen Dent 2005;53:6-9.

15 Tello CG, Ford P, Iacopina AM: In vitro evaluation of complex carbohydrate denture adhesive formulations. Quintessence Int 1998; 29:585-593.

16 Gopikrishna V, Baweja PS, Venkateshbabu N et al: Comparison of coconut water, propolis, HBSS, and milk on PDL cell survival. J Endod 2008;34:587-589.

17 Silva FB, Almeida JM, Sousa SM: Natural medicaments in endodontics - a comparative study of the anti-inflammatory action. Braz Oral Res 2004;18:174-179.

18 Hammad HM, Hammad MM, Abdelhadi IN, et al: Effects of topically applied agents on intra-oral wound healing in a rat model: a clinical and histomorphometric study. Int J Dent Hyg 2011;9:9-16.
19 Al-Bayaty FH, Taiyeb-Ali TB, Abdulla MA, et al: Antibacterial effects of Oradex, Gengigel and Salviathymol-n mouthwash on dental biofilm bacteria. Afr J Microbiol Res 2011;5: 636-642.

20 Inuyama Y, Kitamura C, Nishihara T, et al: Effects of hyaluronic acid sponge as a scaffold on odontoblastic cell line and amputated dental pulp. J Biomed Mater Res B Appl Biomater 2010;92:120-128.

21 Bogović A, Nižetić J, Galić N, et al: The effects of hyaluronic acid, calcium hydroxide, and dentin adhesive on rat odontoblasts and fibroblasts. Arh Hig Rada Toksikol 2011;62: 155-161.

22 Ferraz CC, Gomes BP, Zaia AA, et al: In vitro assessment of the antimicrobial action and the mechanical ability of chlorhexidine gel as an endodontic irrigant. J Endod 2011;27:452455 .

23 Ferraz CC, Gomes BP, Zaia AA, et al: Comparative study of the antimicrobial efficacy of chlorhexidine gel, chlorhexidine solution and sodium hypochlorite as endodontic irrigants. Braz Dent J 2007;18:294-298. 